\title{
BMJ Open Cognitive-behavioral therapy-based intervention to treat symptoms of anxiety in pregnancy in a prenatal clinic using non-specialist providers in Pakistan: design of a randomised trial
}

\author{
Pamela J Surkan (D) , ${ }^{1}$ Syed Usman Hamdani, ${ }^{2,3}$ Zill-e Huma, ${ }^{2}$ Huma Nazir, ${ }^{2}$ \\ Najia Atif, ${ }^{2}$ Armaan A Rowther, ${ }^{1}$ Rizwana Chaudhri, ${ }^{4}$ Shamsa Zafar, ${ }^{2,5}$ \\ Luke C Mullany, ${ }^{1}$ Abid Malik, ${ }^{2,3}$ Atif Rahman ${ }^{6}$
}

To cite: Surkan PJ, Hamdani SU, Huma Z, et al. Cognitive-behavioral therapy-based intervention to treat symptoms of anxiety in pregnancy in a prenatal clinic using non-specialist providers in Pakistan: design of a randomised trial. BMJ Open 2020;10:e037590. doi:10.1136/ bmjopen-2020-037590

- Prepublication history and additional material for this paper are available online. To view these files, please visit the journal online (http://dx.doi. org/10.1136/bmjopen-2020037590).

$\mathrm{AM}$ and $\mathrm{AR}$ contributed equally.

AM and AR are joint last authors.

Received 08 February 2020

Revised 04 March 2020

Accepted 05 March 2020

Check for updates

(C) Author(s) (or their employer(s)) 2020. Re-use permitted under CC BY-NC. No commercial re-use. See rights and permissions. Published by BMJ.

For numbered affiliations see end of article.

Correspondence to Dr Pamela J Surkan; psurkan@jhu.edu

\section{ABSTRACT}

Introduction Prenatal anxiety is a prevalent condition that is harmful for women and a strong predictor of postpartum depression. This trial assesses an intervention initiated in early pregnancy to mid pregnancy among women with clinical or subclinical symptoms of anxiety in Pakistan. Methods and analysis Happy Mother, Healthy Baby (HMHB) is a phase three, two-arm, single-blind, individual randomised clinical trial conducted in the outpatient department of Holy Family Hospital, a large public tertiary care facility affiliated with Rawalpindi Medical University (RMU). Pregnant women (enrolled at $\leq 22$ weeks of gestation) receive six individual HMHB sessions based on cognitive-behavioral therapy (CBT) and relaxation techniques that are administered by non-specialist providers and tailored to address anxiety symptoms. Two to six booster sessions are given between the fifth consecutive weekly core session and the sixth core session that occurs in the third trimester. Apart from baseline data, data are collected in the third trimester, at birth and at 6-weeks postpartum. Primary outcomes include diagnoses of postpartum common mental disorders. Secondary outcomes include symptoms of anxiety and of depression, and birth outcomes including small-for-gestational age, low birth weight and preterm birth. An economic analysis will determine the cost effectiveness of the intervention.

Ethics Ethics approval was obtained from the Johns Hopkins Bloomberg School of Health Institutional Review Board (Baltimore, USA), the Human Development Research Foundation Ethics Committee (Islamabad, Pakistan), the RMU Institutional Research Forum (Rawalpindi, Pakistan) and the National Institute of Mental Health-appointed Global Mental Health Data Safety and Monitoring Board. Dissemination Results from this trial will build evidence for the efficacy of a CBT-based intervention for pregnant women delivered by non-specialised providers. Identification of an evidence-based intervention for anxiety starting in early pregnancy to mid pregnancy may be transferable for use and scale-up in other low-income and middle-income countries.

Trial registration number NCT03880032.
Strengths and limitations of this study

- Selection of an evidence-based approach and adaptation of the intervention was based on extensive formative research with pregnant Pakistani women and their providers.

- A strength of the design includes the exclusion of women who are prenatally depressed, which will allow us to isolate the effects of prevention of symptoms of anxiety.

- A challenge in study implementation includes the continued participation of women through the completion of the intervention and to the final assessment point at 6-week postpartum.

- Given the hospital-based nature of this study, participants do not necessarily represent all women of reproductive age with anxiety, such as rural or other women who tend to give birth at home.

\section{INTRODUCTION}

Pregnant women frequently suffer from common mental health disorders (CMDs) such as anxiety and depression. ${ }^{1}$ In low/ middle-income countries (LMICs), women are particularly predisposed to CMDs, and these disorders are associated with risk factors such as gender discrimination, interpersonal violence, lack of support, poverty and poor health. ${ }^{2}$ In Pakistan, $35 \%^{3}-49 \%{ }^{4}$ of pregnant women suffer from anxiety. These symptoms tend to persist and strongly predict maternal depression and anxiety disorders postpartum, ${ }^{5-9}$ adversely affecting caregiving. ${ }^{10-14}$

Along with significant human and economic costs to mothers, ${ }^{2}$ prenatal CMDs are associated with poor pregnancy outcomes ${ }^{15-18}$ and long-term cognitive and developmental problems in offspring. ${ }^{2}$ 18-23 Thus, preventive efforts targeting CMDs early in the prenatal 
period have enormous potential for reducing the negative effects of postpartum mental disorders on mothers and improving child outcomes. In LMICs, a striking gap exists between mental health needs and access to care. ${ }^{2425}$ At the same time, no current prevention interventions address prenatal anxiety in these settings. Its high prevalence, coupled with limited resources for treatment, results in an urgent need to expand the evidence base for preventive strategies.

While prenatal depression has been the focus of most research on CMD in pregnancy to date, anxiety and depression often co-occur, ${ }^{26}$ and prenatal anxiety (especially occurring early in pregnancy) has been relatively neglected. Exposure to anxiety, even as early as during the second trimester of pregnancy, is important to intrauterine fetal growth. ${ }^{27}$ Given the high prevalence of adverse birth outcomes in Pakistan (eg, $47 \%$ are born small-for-gestational age (SGA)), ${ }^{29}$ this setting provides a unique research opportunity. Due to a paucity of nonpharmaceutical initiatives to address anxiety during pregnancy, our proposed intervention engages nonspecialist providers to target symptoms of anxiety using strategies based on cognitive-behavioral therapy (CBT). CBT is known to effectively treat $\mathrm{CMDs}^{30-32}$ but has been underused for prevention in both high-income and lowincome countries despite evidence-based recommendations. ${ }^{33}$ As both clinical and subthreshold prenatal anxiety strongly predict postpartum depression, ${ }^{6} 7$ anxiety-focused interventions may have carry-over benefits on both depression as well as anxiety in the postpartum period. By creating and testing an intervention to be initiated in the first half of pregnancy, we will provide critically needed evidence on the effectiveness of these approaches to reduce the prevalence of both of these damaging maternal conditions and prevent their collateral effects on newborns. Specifically, we aim to examine the effects of the intervention on postpartum CMDs, birth outcomes and healthcare utilisation costs.
In this manuscript, we give an overview of the design and implementation of this randomised controlled trial (RCT) with the goal of describing its aims, recruitment, randomisation, intervention delivery procedures and analysis plan.

\section{METHODS AND ANALYSIS}

The purpose of this facility-based RCT in Rawalpindi, Pakistan, is to evaluate the impact of using an individually delivered CBT-based approach in a setting with relatively high rates of postpartum CMDs and adverse birth outcomes, for example, SGA and preterm birth (PTB). By targeting pregnant women with subthreshold anxiety while simultaneously including women with high anxiety levels, we aim to measure its effectiveness at reducing postpartum CMDs, using both diagnostic and symptom measures. The primary endpoint of the study is a 6-week postpartum evaluation. A secondary aim is to evaluate the effect of the intervention on birth outcomes including low birth weight (LBW), SGA and PTB. An important feature of the study design is that we exclude women with a current major depressive episode (MDE) in order to gauge the utility of our intervention specifically focused on treating prenatal anxiety symptoms. We will implement the intervention using female non-specialist providers and integrate the programme into a tertiary care setting.

Additional study goals are to examine mediators and moderators of the effects of the intervention (eg, to examine the role of perceived stress, social support, marital relationship and empowerment; figure 1) as well as carry out a cost-effectiveness analysis to ascertain whether participation results in reductions in healthcare usage and costs. We will use the Client Service Receipt Inventory (CSRI) at third trimester and at the 6 weeks' postpartum to collect data on the utilisation and costs of healthcare and loss of productivity (including that of caregivers) from conception to 6-week postnatal. We will

Figure 1 Conceptual model of the Happy Mother-Healthy Baby Study. CMD, common mental health disorder; LBW, low birth weight; PTB, preterm birth; SGA, small-for-gestational age 
collect information about service utilisation and the outof-pocket expenditures of the participants and the infants, including costs for seeing a doctor or other healthcare providers, admission to the hospital, medicines, tests and extra help at home. The CSRI has been used in Pakistan, and an adapted version will be used based on an international study on perinatal depression. ${ }^{34}$

\section{Study overview}

Happy Mother, Healthy Baby (HMHB) is a phase three, twoarm, single-blind, individual randomised clinical trial conducted in the outpatient department of Holy Family Hospital (HFH), a large public tertiary care facility affiliated with Rawalpindi Medical University (RMU).

HMHB is a psychosocial intervention based on CBT for expectant women experiencing anxiety during early pregnancy to mid pregnancy. It draws on the core principles and strategies of the Thinking Healthy Program, an evidence-based psychosocial intervention for mothers experiencing perinatal depression, including psychoeducation, empathetic listening, thought challenging, behaviour activation, family involvement and problem management. ${ }^{35} 36$ Our formative qualitative research immediately prior to the trial indicated the following risk factors for anxiety: adverse life events, interpersonal problems, lack of support, financial problems, lack of appropriate healthcare, gender preference, pregnancy complications and fear of labour (see Atif et $a l^{37}$ for details). These risk factors are consistent with evidence from other studies conducted in Pakistan. ${ }^{938-40}$
The intervention is tailored to address risk factors specific to anxiety during pregnancy that were identified in our formative research. It additionally incorporates stress management skills such as breathing exercises. Culturally relevant customised illustrations are used for guided discovery, behaviour activation, stress management and conveying key health messages. The intervention is a series of 5-weekly one-on-one sessions in early to mid pregnancy complemented by take-home exercisestable 1 . These are accompanied by a final sixth therapy session in the third trimester of pregnancy, with between two and six complementary booster sessions occurring before the final core session in the third trimester (table 1). These visits are coordinated with women's routine hospital appointments when possible. The number of booster sessions will depend on the women's need for additional psychological care and how many additional sessions are possible given her date of enrolment in this study.

Women randomised to the control group will receive enhanced usual care (EUC). WHO recommends eight prenatal visits for positive pregnancy experience, ${ }^{41}$ which is the target number of visits for study participants in the EUC control group (depending on their gestational week at enrolment). These visits will involve evaluating health status, discussing any concerns and performing routine examinations consistent with the stage of pregnancy (table 2). Usual care will also be enhanced by medical staff at the hospital receiving additional training from the depression module of Mental Health Gap Action Program ${ }^{42}$ for mental health treatment. Other

Table 1 Description of intervention sessions

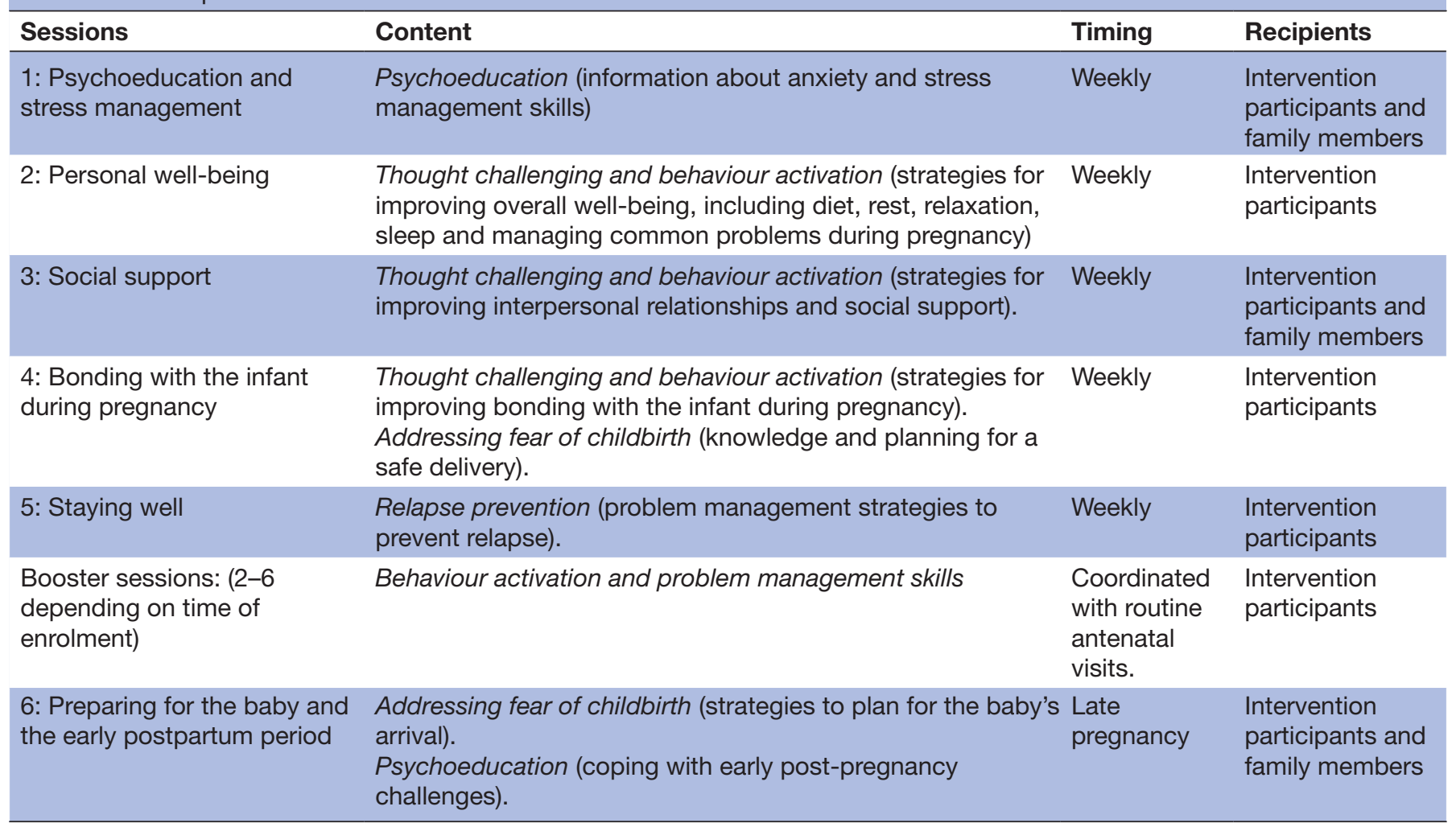




\begin{tabular}{|c|c|c|c|}
\hline Visit & Content & Timing* & Recipients \\
\hline 1 & $\begin{array}{l}\text { Assessment for anaemia; dietary counselling about healthy eating and keeping } \\
\text { physically active during pregnancy; folic acid supplements; counselling for } \\
\text { common physiological symptoms; emphasis on eight antenatal care visits }\end{array}$ & $\begin{array}{l}\text { Following } \\
\text { recruitment }\end{array}$ & $\begin{array}{l}\text { Intervention } \\
\text { and control } \\
\text { participants }\end{array}$ \\
\hline 2 & Same as visit 1 , plus iron supplementation & Second trimester & Same as above \\
\hline 3 & Same as visit 1 , plus iron supplementation & Second trimester & Same as above \\
\hline 4 & Same as visit 1 , plus iron supplementation & Third trimester & Same as above \\
\hline 5 & Same as visit 1 , plus iron supplementation & Third trimester & Same as above \\
\hline 6 & Same as visit 1 , plus iron supplementation & Third trimester & Same as above \\
\hline 7 & Same as visit 1 , plus iron supplementation and tetanus vaccination. & Third trimester & Same as above \\
\hline 8 & Same as visit 1 , plus iron supplementation and tetanus vaccination. & Third trimester & Same as above \\
\hline
\end{tabular}

Ultrasounds will likely be given on 2-3 of these visits, but may be performed at more visits if medically indicated. The timing will be determined by the woman's obstetrician/gynaecologist.

*These correspond to WHO recommended visits.

enhancements to improve adherence to the intervention protocol in both groups include receipt of reimbursements for transportation and for as many ultrasounds as are medically indicated at HFH during pregnancy.

\section{Partial patient involvement}

Patients were involved in the study through their participation in qualitative formative research to inform the design of the intervention. ${ }^{37}$ Through a series of interviews with 19 pregnant women at $\mathrm{HFH}$, we asked for their input regarding sources of anxiety and manifestations of anxiety that were addressed in the curriculum. We also incorporated anxious pregnant women's input regarding the most acceptable and culturally appropriate methods for delivery of the intervention. As part of their training, six therapists piloted the six core intervention sessions with 15 pregnant women who fulfilled the study's eligibility criteria. Of these women, five took part in a qualitative focus group in which they provided input, including about the burden of the intervention.

\section{Enrolment and randomisation}

\section{Recruitment}

Recruitment is carried out by a female assessment team based in the outpatient department that describes and explains the 'HMHB study' to all pregnant women who fulfil the initial screening criteria (eg, gestational age, language, intent to reside in the area) and their families visiting the Gynecology and Obstetrics Department. Pregnant women and families who express interest in participation are invited to be screened for eligibility.

\section{Screening procedure}

Research assistants screen all pregnant women who register at the Gynecology and Obstetrics Department within outpatient services at HFH. We are using three levels of inclusion/exclusion screening criteria that occur during the same time point at enrolment (figures 2 and 3 ). For level 1 screening, we determine if the woman is at $\leq 22$ gestational weeks of pregnancy, age $\geq 18$ years old, resides $\leq 20 \mathrm{~km}$ from $\mathrm{HFH}$ and understands Urdu. If fulfilling these criteria, women expressing initial interest are asked to provide informed consent for completing the next stage of screening (online supplementary file $1)$. At level 2 screening, we ascertain if women have (1) life-threatening health conditions including active severe depression or suicidal ideation, (2) self-report of past or current significant learning disability, (3) self-report of past or current psychiatric disorder (eg, bipolar disorder or schizophrenia) or psychiatric care (eg, current use of anxiolytic drug and/or other psychotropic drug), (4) medical disorders or severe maternal morbidity that require inpatient management that would preclude participation or (5) intensive care unit admission (not only for assessment). Finally, if not excluded on this basis, potential participants will undergo level 3 screening, which includes assessment for at least mild anxiety on the HADS anxiety questions (ie, score $\geq 8$ ) and absence of a current MDE diagnosis on the Structured Clinical Interview for DSM Disorders (SCID). A full SCID interview is only performed on women scoring $\geq 8$ on the HADS depression questions.

\section{Randomisation}

A random sequence of assignments to the study arms was generated using a pseudo random-number generator and constructed using randomly permuted blocks of size $4,8,12$ and 16 by the trial statistician. The assignment list was printed in order, with each step of the sequence separately stored in opaque envelopes and numbered sequentially with a seven-digit code. When an eligible woman subsequently provides consent for study participation (online supplementary file 2), the next available envelope is pulled and opened, and assignment to intervention or control is recorded.

The assessment team, trial statistician, principal investigator and site principal investigators are masked to the allocation status of study participants. To maintain masking during the trial, intervention and assessment 
All women who attend the

Outpatient Gynaecology and Obstetrics Department

Holy Family Hospital - Prenatal Desk

Level 1: Screening for eligibility
Age $\geq 18$
Pregnancy $\leq 22$ weeks
$\leq 20$ Km of Holy Family Hospital
Urdu speaking

Level 1: Screening for eligibility

$\leq 20 \mathrm{Km}$ of Holy Family Hospital

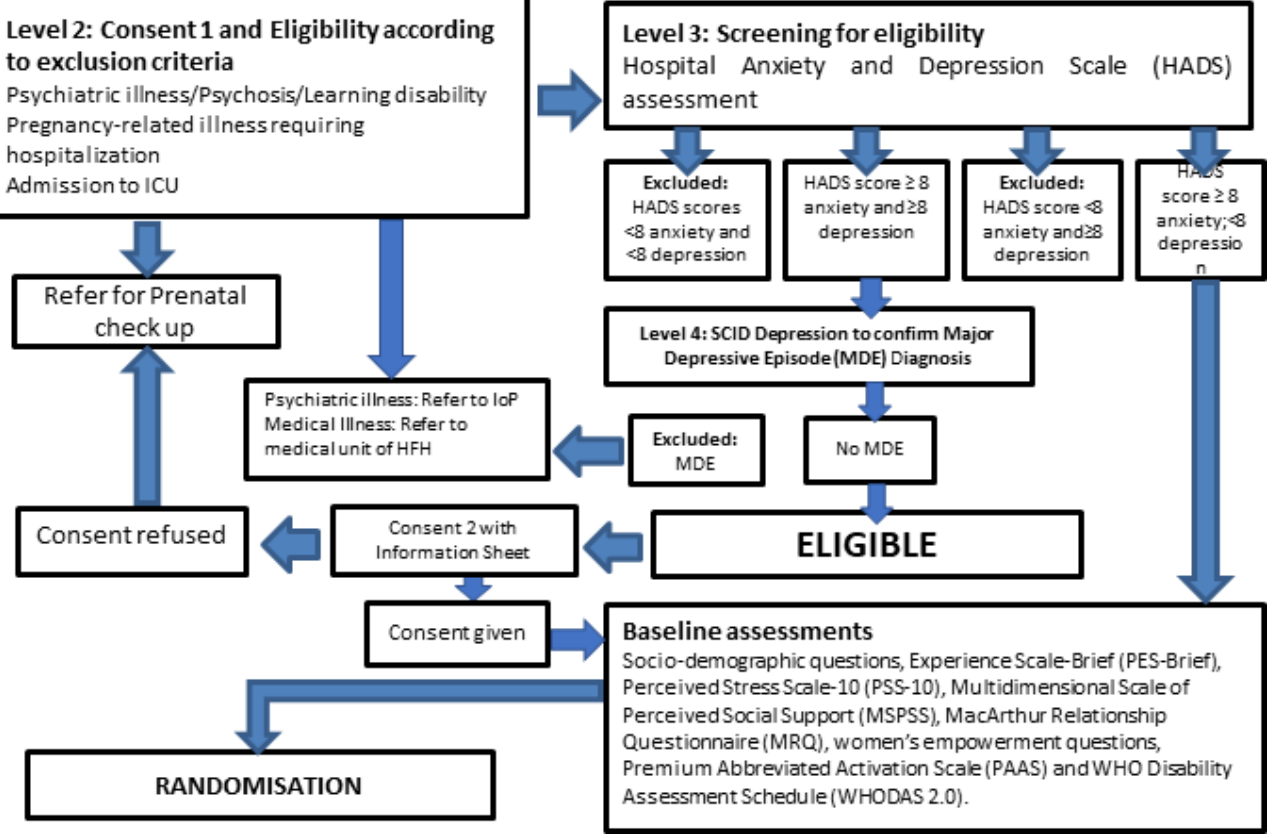

Level 2: Consent 1 and Eligibility according to exclusion criteria

Psychiatric illness/Psychosis/Learning disability

Pregnancy-related illness requiring

hospitalization

Admission to ICU

Figure 2 Screening for eligibility or exclusion and baseline assessments.

teams are stationed at separate locations within the $\mathrm{HFH}$ Gynecology and Obstetrics Department and do not interact during the trial. Participants are also instructed to not disclose which type of treatment they are receiving to the assessment team. Fidelity of masking will be measured by having assessors guess the trial arm of each participant at the end of follow-up assessment. We hypothesise that assessors will only be able to correctly guess the condition of participants at a chance rate of around $50 \%$, indicating that masking is maintained.

\section{Training and supervision of HMHB therapists}

HMHB therapists each have a Bachelor's degree in the social sciences and at least 1 year experience of delivering maternal psychosocial interventions in low-resource settings. Prior to the trial, therapists received 42 hours of classroom training (including presentations, discussions and group activities) delivered by the lead trainer, followed by 2 months of field training. Classroom training focused on understanding anxiety disorder and its impact, use of counselling skills to engage participants, the CBT approach for overcoming anxiety in conjunction with the content and delivery mechanisms of HMHB, and detection and reporting of adverse events. Throughout the training, HMHB therapists were assessed on knowledge and skills required to deliver the intervention using role-playing exercises.

Following the classroom training, all therapists received 2 months of field training to acquire firsthand experience delivering the intervention (under supervision of the master trainer), to at least two expectant women who fulfilled study inclusion criteria. During the field training, therapists delivered the six core sessions to the expectant women.

One-day required refresher trainings will be conducted by the lead trainer twice per year. Refresher training will focus on reviewing content and delivery mechanisms of HMHB and ensuring that therapists retain their competencies, which will be assessed via role-playing exercises.

\section{Supervision of HMHB therapists}

The therapists received weekly group supervision during the pilot phase and continue to receive this supervision during the trial. This supervision is conducted by a psychologist (lead trainer, coinvestigator on this study) in order to provide ongoing support and guidance. Supervision is conducted in-person and online (using online video conferencing software, like Skype).

\section{Assessment of HMHB therapist competence}

To ensure the quality of the intervention and competency of the therapists to provide it, assessments were carried out throughout the training and continue into the trial period. All therapists are evaluated on their knowledge of the intervention (eg, strategies, procedures) and specific skills required to deliver it. These skills are assessed using an adapted version of the ENhancing Assessment of Common Therapeutic factors (ENACT) rating scale ${ }^{43}$ to facilitate rating therapist competence. These assessments will ensure that the intervention is delivered with high enough quality to achieve the expected effects, that all the intervention contents are delivered with fidelity, and that the specific skills required for its delivery are maintained. 
Program or

Enhanced Care

Assessment

Time Point 3

Assessment

Time Point 4

Assessment

Time Point 5

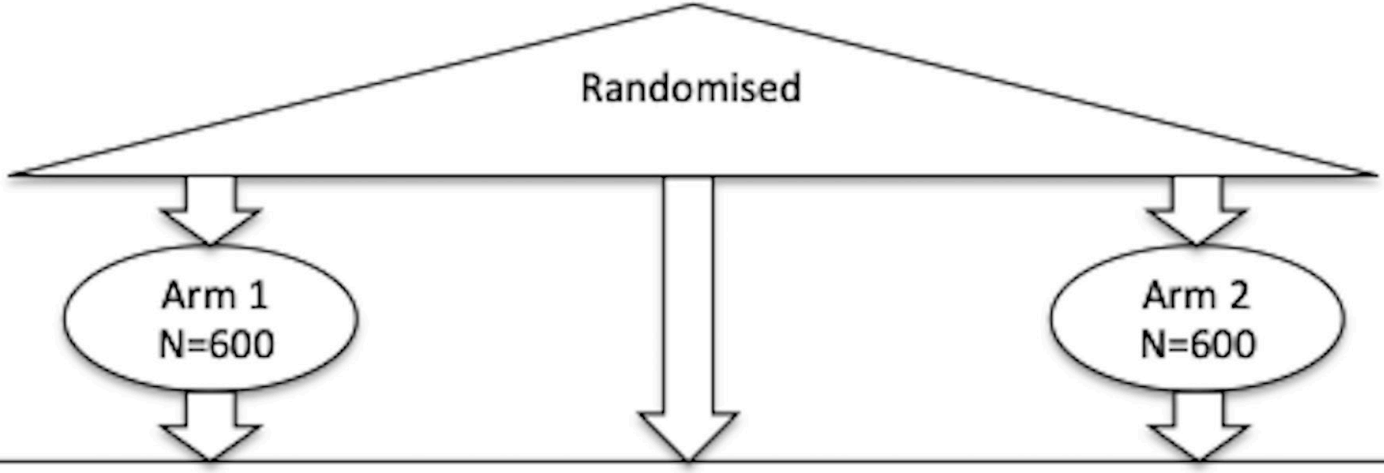

Intervention

All expectant women randomised to the intervention arm will receive 6 sessions plus 2-6 booster sessions of $\mathrm{HMHB}$, and women in the enhanced usual care arm will receive equal number of sessions.

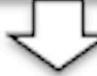

\section{$3^{\text {rd }}$ trimester assessment}

Data will be collected on the PES-Brief, PSS-10, MSPSS, MRQ, WHODAS 2.0, PAAS Client Service Receipt Inventory (CSRI) and women's empowerment questions.

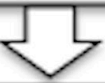

\section{At birth assessment}

SGA at birth, PTB, and LBW. Medical record data on medical condition during pregnancy (pre-eclampsia, gestational diabetes).

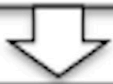

\section{6 weeks' postpartum-end-point assessment}

Data will be collected on child height and weight, medical status of the mother and child, medications in pregnancy, using HADS, SCID for GAD and MDE, Patient Health Questionnaire 9 items (PHQ-9), PSS-10, MSPSS, MRQ, Pakistan DHS IPV questionnaire, WHODAS 2.0, PAAS, CSRI, women's empowerment questions, Breastfeeding Questionnaire, maternal nutrition status (anaemia, MUAC), Postpartum Bonding Questionnaire (PBQ), Maternal Infant Responsiveness Instrument (MIRI), and Maternal SelfEfficacy Questionnaire (MSEQ).

Figure 3 Randomisation and follow-up assessment flow diagram. DHS, Demographic Health Survey; GAD, generalised anxiety disorder; HADS, Hospital Anxiety and Depression Scale; HMHB, Happy Mother, Healthy Baby; IPV, intimate partner violence; LBW, low birth weight; MDE, major depressive episode; MRQ, MacArthur Relationship Questionnaire; MUAC, midupper arm circumference; MSPSS, Multidimensional Scale of Perceived Social Support; PAAS, Premium Abbreviated Activation Scale; PES-Brief, Pregnancy Experience Scale-Brief; PSS-10, Perceived Stress Scale; PTB, preterm birth; SCID, Structured Clinical Interview for DSM Disorders; SGA, small-for-gestational age; WHODAS 2.0, WHO Disability Assessment Schedule.

During the classroom training and refresher training sessions, assessments will be conducted via role-playing exercises. The therapists will be given different scenarios for role-playing exercises, which are assessed by the lead trainer and other HMHB therapists. During the field training, an independent assessor (ie, research assistants who received full $\mathrm{HMHB}$ training as well as training on assessment using the adapted ENACT rating scale) 
observed two randomly selected sessions conducted by each therapist. 'Independent' assessors are research team members not involved in delivering the training or intervention and therefore can conduct unbiased assessments. Therapists are also not involved in selection of the sessions to be rated. The independent assessor reports the results of the evaluation to the supervisor (master trainer), who provides feedback to the therapist. Over the duration of the trial, an independent assessor will randomly observe $15 \%$ of the core sessions (240 sessions) delivered to the participants. The participants are asked permission for the session to be observed, and on receiving permission, an independent assessor evaluates the session and shares the outcome with the supervisor.

\section{Data collection}

Data are being collected at four time points: baseline (at enrolment), in the third trimester, at birth and at 6-week postpartum (see figure 2). Data collection is coordinated with patients' scheduled prenatal visits when possible and takes place at the hospital for the baseline and third trimester visit. Data on birth outcomes are collected via medical records. The final postpartum follow-up will be conducted in the woman's home.

\section{Quantitative endpoints and data analysis}

Our primary endpoint is diagnosis of CMDs, namely generalised anxiety disorder (GAD) and MDE, as assessed by the SCID at 6 -week postpartum. We hypothesise that randomisation of women with subclinical to clinical levels of prenatal anxiety to the intervention arm will result in fewer cases of CMDs at 6-week postpartum compared with the control arm of mothers with similar levels of baseline anxiety who do not receive the intervention. We will also assess women's symptoms of anxiety and depression as endpoints.

Our secondary outcomes include birth outcomes; we hypothesise that randomisation of women with subclinical to clinical levels of prenatal anxiety to the intervention will improve these outcomes, that is, reduce the likelihood of growth restriction (SGA), LBW and PTB.

\section{Sample size determination}

In our study, the unit of randomisation is an individual woman, with an equal number of women randomised to the intervention and control groups. We assumed a significance level of 0.05 . Sample size calculations were based on $\chi^{2}$ tests comparing outcome rates in the intervention and control groups. We assumed an outcome prevalence of CMDs (MDE and GAD) at 30\%, which we considered conservative given that most estimates of prenatal depression in Pakistan are higher than this. ${ }^{34} 3944$ Assuming this prevalence and considering a $30 \%$ reduction in CMDs as meaningful, ${ }^{145}{ }^{46}$ we will need to measure this outcome in 840 pregnant women (420 in each arm) to achieve $85 \%$ power. Based on our prior research experience in Pakistan, we will overenrol to compensate for $30 \%$ attrition post enrolment; this attrition rate will require us to enrol 600 women per group in this study, for a total of 1200 women.

This sample size of 420 outcomes per group provides ample power to detect important reductions in SGA, which we currently estimate to occur in approximately $47 \%$ of births. For example, we will have $90 \%$ and $80 \%$ power to detect reductions in SGA incidence of $23.7 \%$ and $20.2 \%$, respectively. With the sample size of 420 per group, we will have about $85 \%$ power to detect a $21.6 \%$ relative reduction in SGA, if SGA in the population is $47 \%$.

\section{Data management}

Data from participants as well as evaluations of therapists are being collected using the Open Data Kit (ODK) platform on password protected tablets. In preparation for the trial, a full-day workshop was conducted by the study's data manager for training on the use of ODK. A running audit trail is being maintained, in which data are continuously evaluated for outliers by the data collection team and trial statistician. ODK forms, which include all measures assessed including ENACT, are designed to autosave and are sent to the server via the internet at the end of each working day, after which all they are erased from the tablets on which the data are collected. Daily backups are made on a personal computer and the cloud, and weekly backups are made on a password-protected local portable hard drive. The data manager shares a weekly report with investigators in the US and Pakistan including an enrolment table with screening details of the trial participants, information about dropout and any adverse events. Data cleaning takes place at the end of each day. The data manager uses the women's last menstrual period recorded at screening to predict the start of third trimester and generate follow-up lists for the sixth required study visit to take place in the third trimester.

\section{Statistical analyses}

Initial analyses will compare baseline characteristics of research participants between the two study arms; participants who completed follow-up assessments and participants who could not complete follow-up assessments; and the distribution of potential confounding factors. Findings will be reported as per Consolidated Standards of Reporting Trials guidelines for RCTs including a trial flow chart. This will include the flow of participants through each stage of the trial, including the number eligible, randomly assigned, receiving the intended treatment, completing the study protocol and analysed for the primary outcome.

The outcome measures will be summarised at baseline and follow-up, both overall and by intervention arm. These will be summarised by means (SD), medians (IQR) or numbers and proportions as appropriate. For continuous outcomes, histograms within each arm will be plotted to assess how closely the scales follow a normal distribution and to determine how to describe the outcomes and how to properly perform the inferential analysis. 


\section{Analysis of the primary efficacy endpoint}

The primary outcome of the study is the prevalence of cases of CMDs (GAD and MDE) in both study arms. Diagnosed cases of both GAD and MDE will be assessed with the SCID.

All randomised participants for whom we have outcome data will be included in the intention-to-treat analysis. The intervention group (enrolled after being screened for subclinical or clinical levels of anxiety) will be compared with non-intervention controls (selected on the same criteria) to examine the effects of the intervention on development of CMDs. The primary analysis will involve outcome assessment in the mothers randomly assigned to the two conditions using Fisher's exact test. Primary analyses will include direct estimation of the change in mean estimate and/or relative risk of the outcomes along with the $95 \% \mathrm{CI}$ around that outcome using linear and Poisson regression to control for baseline maternal characteristics. ${ }^{47}$ Other sensitivity analyses may evaluate whether there was more of an effect among women who received the full intervention. Variable transformations (such as square root or $\log$ ) will be used as appropriate to meet the model assumptions.

\section{Analysis of secondary endpoints}

We will secondarily compare the intervention and control groups in terms of changes in CMD symptom scores, by measuring depressive symptoms with the nine-item Patient Health Questionnaire and the HADS anxiety questionnaire. Other variables of interest, but not primary targets, include birth outcomes (SGA, LBW and PTB), perceived stress (using the Perceived Stress Scale), pregnancy-related anxiety (using the Pregnancy Experience Scale (PES-Brief)), maternal functioning (using WHO Disability Assessment Schedule), marital relationships (using the MacArthur Relationship Questionnaire), intimate partner violence, women's empowerment, maternal bonding, maternal responsiveness, child growth at 6-week postpartum, as well as initiation and exclusivity of breast feeding (WHO measures). Pregnancy-related symptoms of anxiety (measured with the PES-Brief) will be assessed at baseline and in the third trimester.

For secondary outcomes not related to mental health, we will estimate the effects of the intervention using birth and follow-up (6-week postpartum) measures separately. Main analyses will performed with non-parametric tests, by comparing pre- to post-tests in women randomly assigned to the two conditions; however, sensitivity analyses will use parametric models such as linear and logistic regression to control for baseline values of maternal characteristics. ${ }^{47}$ Variable transformations (such as square root or $\log$ ) will be used as appropriate to meet the model assumptions. For primary and secondary endpoints, in all regression models, the coefficient of the HMHB versus non-HMHB (control group) indicator variable will be taken as the estimated effect.

\section{Economic analysis}

The data collected through the CSRI will be used to calculate service costs and total costs of care for each participant. Unit costs of services itemised in the CSRIsuch as cost per outpatient visit-will be based on locally conducted health facility costing exercises. Service cost data will subsequently be linked to primary and secondary study outcomes to assess issues around the value or cost effectiveness of the task-shifting intervention. In the event that dominance is not shown, that is, the intervention is more effective but the costs in the intervention group are also more than in the EUC group, incremental cost-effectiveness ratios will be computed, together with their CIs (using bootstrapping techniques to overcome the expected skewness of the cost data). Results will be plotted on a cost-effectiveness plane and presented as cost-effectiveness acceptability curves to show the probability of the intervention being cost effective at a range of willingness-to-pay threshold levels. A sensitivity analysis will be conducted to take into account uncertainty and imprecision in the measurements, including multiple imputation models for missing values.

\section{Missing data}

Adjusted analysis and subgroup analysis will be based on covariates at baseline with non-missing values. Sensitivity analysis will be performed on the covariates with imputed missing values. ${ }^{48}$ All analyses will be detailed in the statistical analysis plan, which will be finalised before unblinding the study.

\section{Qualitative process evaluation}

To supplement our quantitative data analysis, we will collect qualitative data from approximately 20 women in the intervention group and 10 women in the EUC group (or until saturation is achieved) to gain an in-depth understanding of the women's experiences with the programme (including opportunity/emotional costs of participation). These women will be purposively sampled to achieve a wide range of variation (or maximum variation) on dimensions of interest (eg, type of stressor identified in the intervention, age, number of prior pregnancies, HADS score). To triangulate and learn from multiple perspectives, we will also hold one focus group discussion with the HMHB therapists.

\section{Ethics}

This study was reviewed and approved by the Johns Hopkins Bloomberg School of Health (JHSPH) Institutional Review Board (Baltimore, USA), the Human Development Research Foundation Ethics Committee (Islamabad, Pakistan), the RMU Institutional Research Forum (Rawalpindi, Pakistan) and the National Institute of Mental Health (NIMH) appointed Global Mental Health Data Safety and Monitoring Board (DSMB).

Women are discontinued from the study in the case of stillbirth or infant death prior to the final assessment at 
6-week postpartum or on request of the participant. No interim analyses or stopping rules are planned.

This study is monitored with oversight by the NIMH DSMB. The committee meets twice a year and is composed of senior global mental health professionals. Detailed reports are sent to the DSMB triannually, including reports on any adverse events. Progress reports are sent to the JHSPH on an annual basis.

\section{DISSEMINATION}

The HMHB Trial is designed to reduce symptoms of anxiety during pregnancy, which has the potential to impact development of maternal postpartum CMDs, including postpartum depression and anxiety as well as intrauterine growth, reflected in SGA, LBW and PTB. Additional findings from this study will describe the mechanism through which the intervention may be operating, particular subgroups for whom the intervention may be more beneficial, as well cost effectiveness of the intervention in terms of costs to both the women and the health system.

The principal investigator and coinvestigators both in the USA and the host country will be given access to the cleaned data sets. Deidentified project data from the study will be posted on clinicaltrials.gov and the NIMH data archive. Study results will be disseminated through peer-reviewed journals and conferences. Data collection for the trial started on April 16, 2019 and will continue until September 2021.

\section{Author affiliations}

1Department of International Health, Johns Hopkins University Bloomberg School of Public Health, Baltimore, Maryland, USA

${ }^{2}$ Human Development Research Foundation, Rawalpindi, Pakistan

${ }^{3}$ Institute of Psychiatry, Rawalpindi Medical University, Rawalpindi, Pakistan

${ }^{4}$ Department of Gynaecology and Obstetrics, Holy Family Hospital, Rawalpindi Medical University, Rawalpindi, Pakistan

${ }^{5}$ Department of Obstetrics and Gynaecology, Air University, Islamabad, Pakistan

${ }^{6}$ Department of Psychological Sciences, University of Liverpool, Liverpool, UK

\section{Twitter Abid Malik @doctorabidmalik}

Contributors PS drafted the original manuscript. SUH, Z-eH, HN, NA, AAR, RC, SZ, $\mathrm{LCM}, \mathrm{AM}$ and $\mathrm{AR}$ contributed to the writing. All authors approved of the final version of the manuscript.

Funding This work was supported by National Institute of Mental Health at the US National Institutes of Health grant number R01 MH111859-01.

\section{Competing interests None declared.}

Patient and public involvement Patients and/or the public were involved in the design, or conduct, or reporting, or dissemination plans of this research. Refer to the Methods section for further details.

\section{Patient consent for publication Not required.}

Provenance and peer review Not commissioned; peer reviewed for ethical and funding approval prior to submission.

Open access This is an open access article distributed in accordance with the Creative Commons Attribution Non Commercial (CC BY-NC 4.0) license, which permits others to distribute, remix, adapt, build upon this work non-commercially, and license their derivative works on different terms, provided the original work is properly cited, appropriate credit is given, any changes made indicated, and the use is non-commercial. See: http://creativecommons.org/licenses/by-nc/4.0/.
ORCID iD

Pamela J Surkan http://orcid.org/0000-0002-0334-5931

\section{REFERENCES}

1 Fisher J, Cabral de Mello M, Patel V, et al. Prevalence and determinants of common perinatal mental disorders in women in low- and lower-middle-income countries: a systematic review. Bull World Health Organ 2012;90:139-49.

2 Wachs TD, Black MM, Engle PL. Maternal depression: a global threat to children's health, development, and behavior and to human rights. Child Dev Perspect 2009;3:51-9.

3 Niaz S, Izhar N, Bhatti MR. Anxiety and depression in pregnant women presenting in the OPD of a teaching hospital. Pak J Med Sci 2004;20:117-9.

4 Waqas A, Raza N, Lodhi HW, et al. Psychosocial factors of antenatal anxiety and depression in Pakistan: is social support a mediator? PLoS One 2015;10:e0116510.

5 Coelho HF, Murray L, Royal-Lawson M, et al. Antenatal anxiety disorder as a predictor of postnatal depression: a longitudinal study. $J$ Affect Disord 2011;129:348-53.

6 Norhayati MN, Hazlina NHN, Asrenee AR, et al. Magnitude and risk factors for postpartum symptoms: a literature review. J Affect Disord 2015;175:34-52.

7 Robertson E, Grace S, Wallington T, et al. Antenatal risk factors for postpartum depression: a synthesis of recent literature. Gen Hosp Psychiatry 2004;26:289-95.

8 Sutter-Dallay AL, Giaconne-Marcesche V, Glatigny-Dallay E, et al. Women with anxiety disorders during pregnancy are at increased risk of intense postnatal depressive symptoms: a prospective survey of the MATQUID cohort. Eur Psychiatry 2004;19:459-63.

9 Husain N, Bevc I, Husain M, et al. Prevalence and social correlates of postnatal depression in a low income country. Arch Womens Ment Health 2006;9:197-202.

10 Field T. Postpartum depression effects on early interactions, parenting, and safety practices: a review. Infant Behav Dev 2010;33:1-6.

11 McLearn KT, Minkovitz CS, Strobino DM, et al. The timing of maternal depressive symptoms and mothers' parenting practices with young children: implications for pediatric practice. Pediatrics 2006;118:e174-82.

12 Rahman A, Hafeez A, Bilal R, et al. The impact of perinatal depression on exclusive breastfeeding: a cohort study. Matern Child Nutr 2016;12:452-62.

13 Hurley KM, Black MM, Papas MA, et al. Maternal symptoms of stress, depression, and anxiety are related to nonresponsive feeding styles in a statewide sample of WIC participants. J Nutr 2008;138:799-805.

14 Lovejoy MC, Graczyk PA, O'Hare E, et al. Maternal depression and parenting behavior: a meta-analytic review. Clin Psychol Rev 2000;20:561-92.

15 Grote NK, Bridge JA, Gavin AR, et al. A meta-analysis of depression during pregnancy and the risk of preterm birth, low birth weight, and intrauterine growth restriction. Arch Gen Psychiatry 2010;67:1012-24.

16 Dunkel-Schetter C. Stress in pregnancy: emperial evidence and theoretic al issues to guide interdisciplinary researchers. In: Handbook of stress science: biology, psychology, and health. New York: Springer Publishing Company, 2011: 321-43.

17 Dunkel-Schetter C, Lobel M. Pregnancy and birth: A multilevel analysis of stress and birthweight. In: Revenson T, Baum A, Singer J, eds. Handbook of health psychology. Mahwah NJ: Lawence Erlbaum, 2011: 427-53.

18 Surkan PJ, Patel SA, Rahman A. Preventing infant and child morbidity and mortality due to maternal depression. Best Pract Res Clin Obstet Gynaecol 2016;36:156-68.

19 Talge NM, Neal C, Glover V, et al. Antenatal maternal stress and long-term effects on child neurodevelopment: how and why? J Child Psychol Psychiatry 2007;48:245-61.

20 Seckl JR. Glucocorticoids, developmental 'programming' and the risk of affective dysfunction. Prog Brain Res 2008;167:17-34.

21 Tarabulsy GM, Pearson J, Vaillancourt-Morel M-P, et al. MetaAnalytic findings of the relation between maternal prenatal stress and anxiety and child cognitive outcome. J Dev Behav Pediatr 2014;35:38-43.

22 O'Connor TG, Heron J, Glover V, et al. Antenatal anxiety predicts child behavioral/emotional problems independently of postnatal depression. J Am Acad Child Adolesc Psychiatry 2002;41:1470-7.

23 Zelkowitz P, Papageorgiou A. Easing maternal anxiety: an update. Womens Health 2012;8:205-13. 
24 Saxena S, Thornicroft G, Knapp M, et al. Resources for mental health: scarcity, inequity, and inefficiency. Lancet 2007;370:878-89.

25 Demyttenaere K, Bruffaerts R, Posada-Villa J, et al. Prevalence, severity, and unmet need for treatment of mental disorders in the world Health organization world mental health surveys. JAMA 2004;291:2581-90.

26 Pollack MH. Comorbid anxiety and depression. J Clin Psychiatry 2005;66:22-9.

27 Khashan AS, Everard C, McCowan LME, et al. Second-Trimester maternal distress increases the risk of small for gestational age. Psychol Med 2014;44:2799-810.

28 Class QA, Lichtenstein P, Långström N, et al. Timing of prenatal maternal exposure to severe life events and adverse pregnancy outcomes: a population study of 2.6 million pregnancies. Psychosom Med 2011;73:234-41.

29 Lee ACC, Katz J, Blencowe $\mathrm{H}$, et al. National and regional estimates of term and preterm babies born small for gestational age in 138 lowincome and middle-income countries in 2010. Lancet Glob Health 2013;1:e26-36.

30 Cape J, Whittington C, Buszewicz M, et al. Brief psychological therapies for anxiety and depression in primary care: meta-analysis and meta-regression. BMC Med 2010;8:38

31 Green SM, Haber E, Frey BN, et al. Cognitive-Behavioral group treatment for perinatal anxiety: a pilot study. Arch Womens Ment Health 2015;18:631-8.

32 Misri S, Abizadeh J, Sanders S, et al. Perinatal generalized anxiety disorder: assessment and treatment. $J$ Womens Health 2015;24:762-70.

33 The Guideline Development Group - National Collaborating Centre and NICE project team. Antenatal and postnatal mental health: clinical management and service guidance: National Institute for health and care excellence, 2014. Available: https://www.nice.org.uk guidance/cg192 [Accessed 3 Feb 2020].

34 Chisholm D, Conroy S, Glangeaud-Freudenthal N, et al. Health services research into postnatal depression: results from a preliminary cross-cultural study. Br J Psychiatry Suppl 2004;46:s45-52.

35 Rahman A. Challenges and opportunities in developing a psychological intervention for perinatal depression in rural Pakistan-a multi-method study. Arch Womens Ment Health 2007;10:211-9.

36 Rahman A, Malik A, Sikander S, et al. Cognitive behaviour therapybased intervention by community health workers for mothers with depression and their infants in rural Pakistan: a cluster-randomised controlled trial. Lancet 2008;372:902-9.

37 Atif N, Nazir H, Zafar S, et al. Development of a psychological intervention to address anxiety during pregnancy in a low-income country. Front. Psychiatry;10:927.

38 Rahman A, lqbal Z, Harrington R. Life events, social support and depression in childbirth: perspectives from a rural community in the developing world. Psychol Med 2003;33:1161-7.

39 Ali NS, Azam IS, Ali BS, et al. Frequency and associated factors for anxiety and depression in pregnant women: a hospital-based crosssectional study. ScientificWorldJournal 2012;2012:1-9.

40 Humayun A, Haider II, Imran N, et al. Antenatal depression and its predictors in Lahore, Pakistan. East Mediterr Health $\mathrm{J}$ 2013;19:327-32.

41 World Health Organization. WHO recommendations on antenatal care for a positive pregnancy experience. Geneva, Switzerland, 2016.

42 World Health Organization. mhGAP intervention guide for mental, neurological and substance use disorders in non-specialized health settings: mental health gap action programme (ImhGAP) Fversion 2.0, 2016. Available: https://www.who.int/mental_health/ publications/mhGAP_intervention_guide/en/ [Accessed 21 Dec 2019].

43 Kohrt BA, Jordans MJD, Rai S, et al. Therapist competence in global mental health: development of the enhancing assessment of common therapeutic factors (ENACT) rating scale. Behav Res Ther 2015;69:11-21.

44 Karmaliani R, Asad N, Bann CM, et al. Prevalence of anxiety, depression and associated factors among pregnant women of Hyderabad, Pakistan. Int J Soc Psychiatry 2009;55:414-24.

45 Patel V, Weiss HA, Chowdhary N, et al. Effectiveness of an intervention led by lay health counsellors for depressive and anxiety disorders in primary care in Goa, India (MANAS): a cluster randomised controlled trial. Lancet 2010;376:2086-95.

46 Rahman A, Fisher J, Bower P, et al. Interventions for common perinatal mental disorders in women in low- and middle-income countries: a systematic review and meta-analysis. Bull World Health Organ 2013;91:593-601.

47 McCullagh P, Nelder JA, models Glinear, et al. Generalized linear models. New York: CRC Press, 1989.

48 Enders CK. Applied missing data analysis. New York, NY: Guilford Press, 2010. 